\title{
Heavy color-octet particles at the LHC
}

\author{
Chien-Yi Chen, ${ }^{a}$ Ayres Freitas, ${ }^{b}$ Tao Han $^{b}$ and Keith S.M. Lee ${ }^{c, d}$ \\ ${ }^{a}$ Department of Physics, Brookhaven National Laboratory, \\ Upton, NY 11973, U.S.A. \\ ${ }^{b}$ PITTsburgh Particle physics, Astrophysics, and Cosmology Center (PITT PACC), \\ Department of Physics \& Astronomy, University of Pittsburgh, Pittsburgh, PA 15260, U.S.A. \\ ${ }^{c}$ Perimeter Institute for Theoretical Physics, \\ Waterloo, ON N2L 2Y5, Canada \\ ${ }^{d}$ Department of Physics $\&$ Astronomy, University of Waterloo, \\ Waterloo, ON N2L 3G1, Canada \\ E-mail: cychen@bnl.gov, afreitas@pitt.edu, than@pitt.edu, \\ k95lee@uwaterloo.ca
}

ABSTRACT: Many new-physics models, especially those with a color-triplet top-quark partner, contain a heavy color-octet state. The "naturalness" argument for a light Higgs boson requires that the color-octet state be not much heavier than a $\mathrm{TeV}$, and thus it can be pair-produced with large cross sections at high-energy hadron colliders. It may decay preferentially to a top quark plus a top partner, which subsequently decays to a top quark plus a color-singlet state. This singlet can serve as a WIMP dark-matter candidate. Such decay chains lead to a spectacular signal of four top quarks plus missing energy. We pursue a general categorization of the color-octet states and their decay products according to their spin and gauge quantum numbers. We review the current bounds on the new states at the LHC and study the expected discovery reach at the $8-\mathrm{TeV}$ and $14-\mathrm{TeV}$ runs. We also present the production rates at a future $100-\mathrm{TeV}$ hadron collider, where the cross sections will be many orders of magnitude greater than at the 14-TeV LHC. Furthermore, we explore the extent to which one can determine the color octet's mass, spin, and chiral couplings. Finally, we propose a test to determine whether the fermionic color octet is a Majorana particle.

Keywords: Phenomenology of Field Theories in Higher Dimensions, Supersymmetry Phenomenology

ARXIV EPRINT: 1410.8113 


\section{Contents}

1 Introduction 1

2 General framework $\quad 2$

3 Current bounds from the 8-TeV LHC 6

4 Signal observability at the 14-TeV LHC 8

5 Determination of model properties $\quad 9$

$\begin{array}{lll}5.1 \text { Masses } & 10\end{array}$

$\begin{array}{lll}5.2 \text { Spin } & 11\end{array}$

5.3 Couplings 13

5.4 Distinguishing between Majorana and Dirac particles 14

6 Conclusions 16

\section{Introduction}

The historic discovery of the Higgs boson has led particle physics to an interesting juncture. On the one hand, for the first time in history, we have a consistent relativistic quantummechanical model, the Standard Model (SM), that is valid all the way up to the Planck scale. On the other hand, there remain many unanswered theoretical and observational questions, which imply the need for physics beyond the SM. The putative "naturalness" of a light Higgs boson is arguably a strong indication of new physics near the TeV scale, and a top-quark partner is eagerly anticipated as a cure for the quadratic sensitivity of the Higgs mass to the new-physics scale.

Besides the color-triplet top-quark partner, many new-physics models contain a heavy color-octet state. The naturalness argument requires the color-octet state to be not much heavier than the $\mathrm{TeV}$ scale $[1,2]$, which should be accessible at LHC energies (for a recent account, see for example ref. [3] and references therein). Examples of electrically neutral color-octet particles include the gluino in supersymmetry [4], techni-rhos [5] or top-gluons $[6,7]$ in models with strong $\mathrm{TeV}$-scale dynamics, and Kaluza-Klein (KK) gluons in models with universal extra dimensions [8-10]. For large regions of parameter space in these models, the color-octet particles decay preferentially to a top quark plus a heavy top-quark partner, either owing to large couplings between the color octet and the top-quark partner or because other new particles are very massive and thus effectively decoupled. The top partner subsequently decays to a top quark plus a color-singlet state. These decay chains lead to a spectacular signal of four top quarks plus missing energy. 
In this paper, we model-independently study processes of the form

$$
p p \rightarrow \mathcal{Z} \overline{\mathcal{Z}} \rightarrow t \bar{t} Y \bar{Y} \rightarrow t \bar{t} t \bar{t} X \bar{X},
$$

where $\mathcal{Z}$ is a new color-octet particle, $Y$ a new color-triplet particle (top partner), and $X$ a color singlet. The electrically neutral $X$ is assumed to be stable and thus could be a darkmatter candidate, which would manifest itself as missing energy in a collider experiment. For all new particles in this process $(X, Y$ and $\mathcal{Z})$, we consider different spin assignments $(0,1 / 2$ and 1$)$. We also distinguish the possibility that the color octet may or may not be its own antiparticle $(\mathcal{Z}=\overline{\mathcal{Z}}$ or $\mathcal{Z} \neq \overline{\mathcal{Z}})$. Each combination is exemplified by particles in wellmotivated new-physics models. For example, $\mathcal{Z}$ could be the gluino, $Y$ a scalar top, and $X$ the lightest neutralino in the Minimal Supersymmetric Standard Model (MSSM). This case has been studied extensively (see, for example, refs. [3, 11-22]). Vector (scalar) $X$ and $\mathcal{Z}$ particles appear in models with at least one (at least two) universal extra dimension(s) [810], stemming from the KK excitations of the multidimensional gauge-boson fields. ${ }^{1}$ Spin-0 color singlets and octets are also found in $\mathcal{N}=2$ supersymmetry (SUSY) [26-33]. Fermionic and vector top partners, $Y$, exist in extra-dimensional models [8-10] and SUSY models with an extended gauge sector [34]. However, instead of focusing on specific particles in a particular model, we pursue a general categorization in this paper, assuming only a discrete symmetry that ensures the stability of $X$.

Color octets with $\mathcal{O}(\mathrm{TeV})$ masses can be pair produced with large cross sections at the LHC. Consequently, within the framework of the MSSM, the ATLAS and CMS collaborations have put strong bounds on their parameter space [11-18]. In this paper, we recast these limits for different spin assignments of the new particles. Despite these bounds, we show that the full-energy run of the LHC (with $13-14 \mathrm{TeV}$ collision energy) will have a significantly expanded potential for searching for and possibly discovering a signature of the type in eq. (1.1). If a signal is observed, the next goal will be the determination of the spins and couplings of the new particles, $\mathcal{Z}, Y$ and $X$. We study several observables for this purpose and demonstrate their usefulness with numerical Monte Carlo simulation. To avoid ambiguities due to model-dependent branching fractions, we do not rely on the total cross section in this set of variables. Looking further ahead, we also present the cross sections at the 100-TeV VLHC, where the signal production rates can be several orders of magnitude greater than the $14-\mathrm{TeV}$ expectations and thus extend the discovery range substantially.

This paper is organized as follows. After introducing the model-independent classification of gluon and top partners and discussing their production and decay in section 2, we show the current bounds and future reach of the LHC in sections 3 and 4, respectively. Section 5 is devoted to the determination of the masses, spins and couplings of the new particles from LHC data. Finally, conclusions are presented in section 6 .

\section{General framework}

In the following, we study the phenomenology of the Standard Model extended by three new particles: a neutral color singlet $X$, a color triplet $Y$ (and its antiparticle $\bar{Y}$ ) with

\footnotetext{
${ }^{1}$ They can also occur in models with extended gauge groups [23-25].
} 


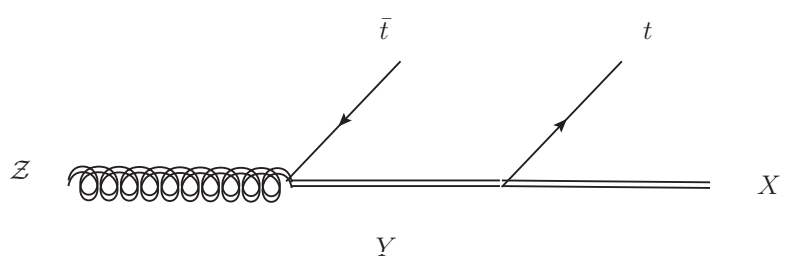

Figure 1. The decay chain of $\mathcal{Z}$ to the color singlet $X$ via the color triplet $Y$. Double lines denote new particles, while single lines denote SM particles. If $\mathcal{Z}$ is a self-conjugate field, this decay chain is accompanied by the charge-conjugate version $\mathcal{Z} \rightarrow t \bar{Y} \rightarrow t \bar{t} X$.
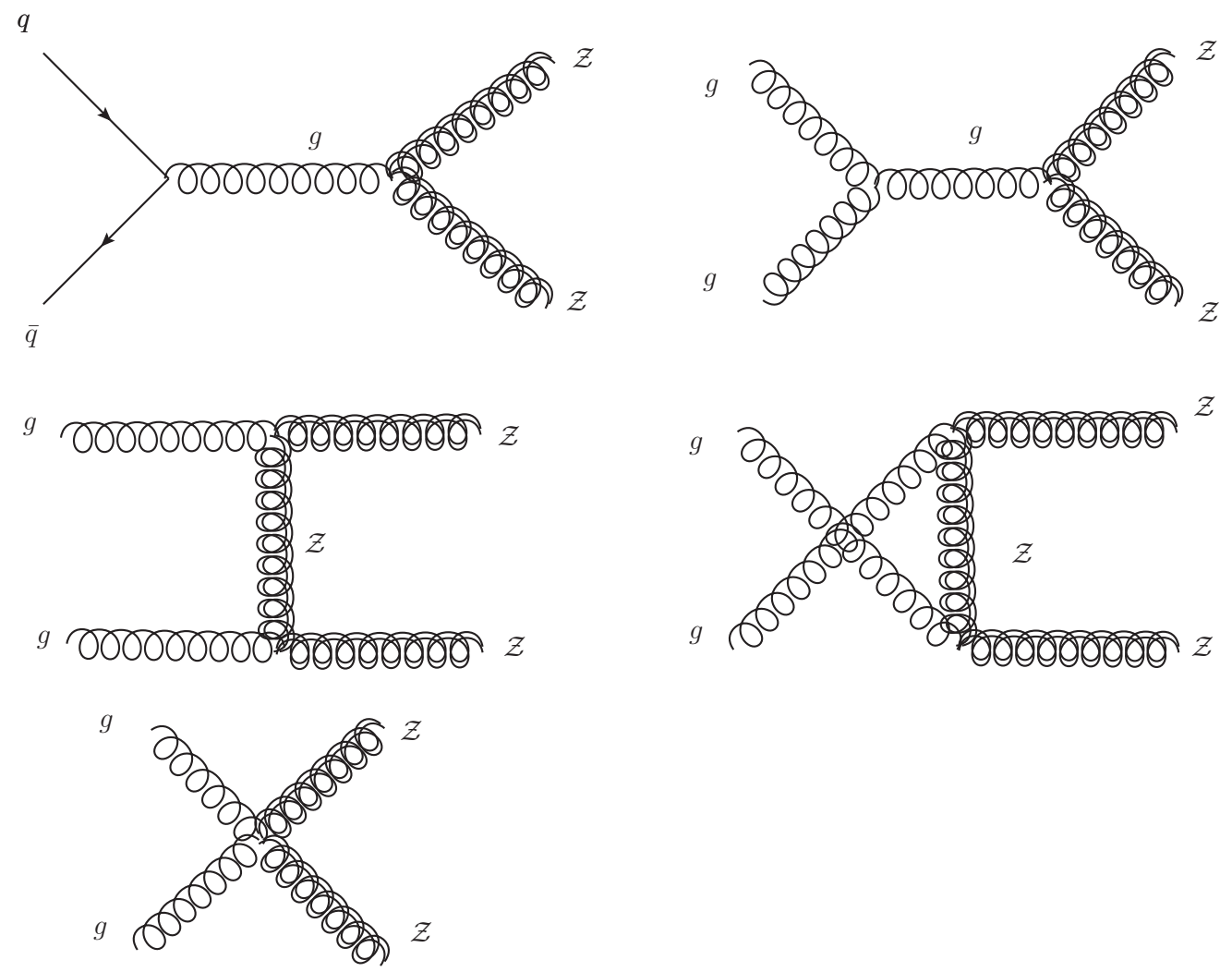

Figure 2. Leading-order diagrams for the pair production of the color octet $\mathcal{Z}$ at hadron colliders. Double lines denote new particles, while single lines denote SM particles. The last diagram exists only for a bosonic $\mathcal{Z}$.

charge $+2 / 3(-2 / 3)$, and a color octet $\mathcal{Z}$. All new particles are assumed to be charged under some new global symmetry, so that they can be produced only in pairs and their decay chains end with the lightest new particle, which, because of astrophysical limits, must be the $X$. Searches for gluinos by ATLAS and CMS [11-18] have led to strong lower bounds on the mass of the $\mathcal{Z}$, so it is reasonable to assume the mass hierarchy $m_{\mathcal{Z}}>m_{Y}+m_{t}$, $m_{Y}>m_{X}+m_{t}$, leading to the decay chain shown in figure 1 .

When one demands gauge invariance and renormalizability, there are four possible spin combinations (with spin $0,1 / 2$ and 1) allowing a coupling between $Y, X$ and $t$, and four combinations for a coupling between $\mathcal{Z}, Y$ and $t$. We summarize the possible combinations 


\begin{tabular}{|c|c|c|c|c|c|c|}
\hline & $\begin{array}{c}Y \\
s, I_{\mathrm{SU}(3)}\end{array}$ & $\begin{array}{c}X \\
s, I_{\mathrm{SU}(3)}\end{array}$ & $\begin{array}{c}G Y Y \\
\text { coupling }\end{array}$ & $\begin{array}{c}X Y t \\
\text { coupling }\end{array}$ & \multicolumn{2}{|c|}{$\begin{array}{l}\text { sample model and decay } \\
Y \rightarrow t X\end{array}$} \\
\hline i & 0,3 & $\frac{1}{2}, \mathbf{1}$ & $G^{a \mu} Y^{*} \overleftrightarrow{\partial_{\mu}} T^{a} Y$ & $\bar{X} \Gamma t Y^{*}$ & MSSM & $\tilde{t} \rightarrow t \tilde{\chi}_{1}^{0}$ \\
\hline ii & $\frac{1}{2}, 3$ & 0,1 & $\bar{Y} \mathscr{G}^{a} T^{a} Y$ & $\bar{Y} \Gamma t X$ & UED & $t_{\mathrm{KK}} \rightarrow t \gamma_{H, \mathrm{KK}}$ \\
\hline iii & $\frac{1}{2}, 3$ & $1, \mathbf{1}$ & $\bar{Y} \phi^{a} T^{a} Y$ & $\bar{Y} X \Gamma t$ & UED & $t_{\mathrm{KK}} \rightarrow t \gamma_{\mathrm{KK}}$ \\
\hline iv & 1,3 & $\frac{1}{2}, \mathbf{1}$ & $S_{3}\left[G, Y, Y^{*}\right]$ & $\bar{X} Y^{*} \Gamma t$ & [34] & $\vec{Q} \rightarrow t \tilde{\chi}_{1}^{0}$ \\
\hline
\end{tabular}

\begin{tabular}{|c|c|c|c|c|c|c|}
\hline & $\begin{array}{c}\mathcal{Z} \\
s, I_{\mathrm{SU}(3)}\end{array}$ & $\begin{array}{c}Y \\
s, I_{\mathrm{SU}(3)}\end{array}$ & $\begin{array}{c}G \mathcal{Z Z} \\
\text { coupling }\end{array}$ & $\begin{array}{c}\mathcal{Z} Y t \\
\text { coupling }\end{array}$ & \multicolumn{2}{|c|}{$\begin{array}{l}\text { sample model and decay } \\
\mathcal{Z} \rightarrow Y \bar{t}\end{array}$} \\
\hline $\mathrm{v}$ & 0,8 & $\frac{1}{2}, 3$ & $G^{a \mu} \mathcal{Z}^{c} \overleftrightarrow{\partial_{\mu}} \mathcal{Z}^{b} f^{a b c}$ & $\bar{Y} T^{a} \Gamma^{\prime} t \mathcal{Z}^{a}$ & UED & $g_{H} \rightarrow t_{\mathrm{KK}} \bar{t}$ \\
\hline vi(a) & $\frac{1}{2}, 8$ & 0,3 & $\overline{\mathcal{Z}}^{c} \mathscr{G}^{a} \mathcal{Z}^{b} f^{a b c}$ & $\overline{\mathcal{Z}}^{a} Y^{*} T^{a} \Gamma^{\prime} t$ & MSSM & $\tilde{g} \rightarrow \tilde{t} \bar{t}$ \\
\hline vi(b) & $\frac{1}{2}, 8$ & 0,3 & $\overline{\mathcal{Z}}_{D}^{c} \mathbb{G}^{a} \mathcal{Z}_{D}^{b} f^{a b c}$ & $\begin{array}{l}\left(\overline{\mathcal{Z}}_{D}^{a}\right)^{*} Y^{*} T^{a} b_{L} t_{L} \\
+\overline{\mathcal{Z}}_{D}^{a} Y^{*} T^{a} b_{R} t_{R}\end{array}$ & $\begin{array}{l}\mathcal{N}=2 \\
\text { SUSY }\end{array}$ & $\tilde{g}_{D} \rightarrow \tilde{t} \bar{t}$ \\
\hline vii & $\frac{1}{2}, 8$ & 1,3 & $\overline{\mathcal{Z}}^{c} \mathscr{G}^{a} \mathcal{Z}^{b} f^{a b c}$ & $\overline{\mathcal{Z}}^{a} Y^{*} T^{a} \Gamma^{\prime} t$ & {$[34]$} & $\tilde{g} \rightarrow \vec{Q} \bar{t}$ \\
\hline viii & 1,8 & $\frac{1}{2}, \mathbf{3}$ & $S_{8}[G, \mathcal{Z}, \mathcal{Z}]$ & $\bar{Y} \not^{a} T^{a} \Gamma^{\prime} t$ & UED & $g_{\mathrm{KK}} \rightarrow t_{\mathrm{KK}} \bar{t}$ \\
\hline
\end{tabular}

$$
\Gamma \equiv a_{L} P_{L}+a_{R} P_{R}, \quad \Gamma^{\prime} \equiv b_{L} P_{L}+b_{R} P_{R}
$$

$$
\begin{aligned}
& A_{\partial_{\mu}} B \equiv A\left(\partial_{\mu} B\right)-\left(\partial_{\mu} A\right) B \\
& S_{3}\left[G, Y, Y^{*}\right] \equiv G_{\mu}^{a} Y_{\nu}^{*} \overleftrightarrow{\partial^{\mu}} T^{a} Y^{\nu}+G_{\mu}^{a} Y^{\mu *} \overleftarrow{\partial^{\nu}} T^{a} Y_{\nu}-G_{\mu}^{a} Y_{\nu}^{*} \overrightarrow{\partial^{\nu}} T^{a} Y^{\mu} \\
& S_{8}\left[G, \mathcal{Z}, \mathcal{Z}^{*}\right] \equiv f^{a b c}\left[G_{\mu}^{a} \mathcal{Z}_{\nu}^{c *} \overleftrightarrow{\partial^{\mu}} \mathcal{Z}^{b \nu}+\mathcal{Z}_{\mu}^{b} G_{\nu}^{a} \overleftrightarrow{\partial^{\mu}} \mathcal{Z}^{c \nu *}+\mathcal{Z}_{\mu}^{c *} \mathcal{Z}_{\nu}^{b} \overleftrightarrow{\partial^{\mu}} G^{a \nu}\right]
\end{aligned}
$$

Table 1. Quantum numbers and couplings of the new particles, $X, Y$ and $\mathcal{Z}$, which interact with the SM top quark, $t$. In the last column, $\tilde{g}, \tilde{t}$ and $\tilde{\chi}_{1}^{0}$ are the gluino, the scalar top and lightest neutralino in the MSSM, respectively [4]. $g_{\mathrm{KK}}, t_{\mathrm{KK}}, \gamma_{\mathrm{KK}}, g_{H}$ and $\gamma_{H, \mathrm{KK}}$ are the first-level KaluzaKlein excitations of the gluon, the top, the photon, and an extra-dimensional component of a gluon and a photon, respectively, in universal extra dimensions (UED) [8-10]. $\tilde{g}_{D}$ denotes a Dirac gluino in $\mathcal{N}=2$ supersymmetry [27, 28, 36]. Finally, $\vec{Q}$ is the vector superpartner in a supersymmetric model with an extended gauge sector [34].

in table 1. The top panel of the table (cases i-iv) reproduces the color-triplet interactions constructed in ref. [35]. These form the basis for our current extended theoretical framework including the color octet $\mathcal{Z}$ (cases v-viii). By default, the $X$ and $\mathcal{Z}$ are assumed to be selfconjugate, but we also explore the phenomenological differences between the cases where $\mathcal{Z}$ is a Majorana fermion (case vi(a)) and a Dirac fermion (case vi(b)), denoted $\mathcal{Z}_{D}$, which can arise in models with $\mathcal{N}=2$ supersymmetry [27, 28]. Also shown in the table are the structure of the relevant couplings and examples of concrete realizations of each case in specific models. For fermions, we allow a general chirality structure, specified by the parameters $a_{L, R}$ and $b_{L, R}$. 

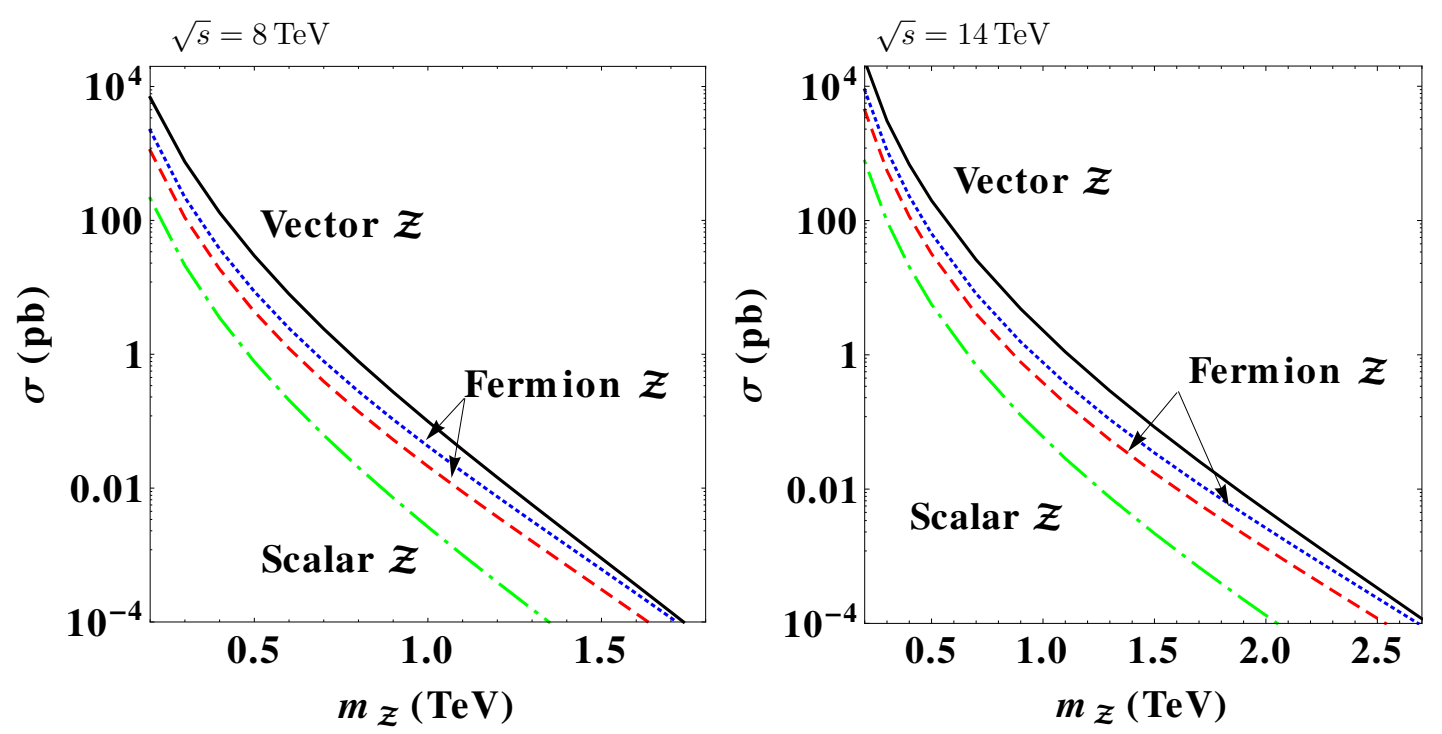

Figure 3. Production cross sections for $p p \rightarrow \mathcal{Z} \overline{\mathcal{Z}}$ at the $\mathrm{LHC}$ for $8 \mathrm{TeV}$ (left) and $14 \mathrm{TeV}$ (right), as a function of the mass $m_{\mathcal{Z}}$, for a vector $\mathcal{Z}$ (black solid), Dirac $\mathcal{Z}$ (blue dotted), Majorana $\mathcal{Z}$ (red dashed), and scalar $\mathcal{Z}$ (green dot-dashed).

Direct production of $Y \bar{Y}$ pairs was discussed in detail in ref. [35]. Here we consider pair production of $\mathcal{Z}$ particles, which subsequently decay according to the decay chain in figure 1. They can be produced with sizeable cross sections at the LHC, even for large masses, $m_{\mathcal{Z}}>1 \mathrm{TeV}$, and lead to a distinct final state of four top quarks and missing energy. The dominant modes for $\mathcal{Z}$ pair production at hadron colliders are the QCD subprocesses

$$
q \bar{q}, g g \rightarrow \mathcal{Z} \overline{\mathcal{Z}}
$$

which are described at leading order by the diagrams in figure 2. The form of the gluon- $\mathcal{Z}$ vertex is dictated by QCD gauge invariance and shown in table 1.

For the different $\mathcal{Z}$ spin assignments, the total QCD cross sections at the LHC are shown in figure 3 as a function of $m_{\mathcal{Z}}$. The values include next-to-leading-order (NLO) QCD corrections for the scalar $\mathcal{Z}$ [37], and NLO and next-to-next-to-leading logarithmic (NNLL) corrections for the Majorana fermion $\mathcal{Z}$ [38], with extrapolation to larger or smaller values of $m_{\mathcal{Z}}$ where necessary. For pair production of color-octet Dirac fermions and vectors, the QCD corrections have not been calculated to the best of our knowledge. Therefore, we simply assume that the $K$-factor for Dirac fermions is identical to the one for Majorana fermions, and that the $K$-factor for the spin- 1 case is the same as for the scalar case, since scalars and vectors share the same diagrams.

As figure 3 shows, the cross section for fermion $\mathcal{Z}$ pairs is about one order of magnitude larger than for the scalar case, because of the fermion's larger number of spin degrees of freedom and $p$-wave suppression of the scalar. The latter effect is most pronounced near threshold, where the $p$-wave production has a velocity dependence of $\sigma \sim \beta^{3}=$ $\left(1-4 m_{\mathcal{Z}}^{2} / \hat{s}\right)^{3 / 2}$, whereas an $s$-wave leads to $\sigma \sim \beta$. As a result, the difference between 
(a) $\sqrt{s}=100 \mathrm{TeV}$

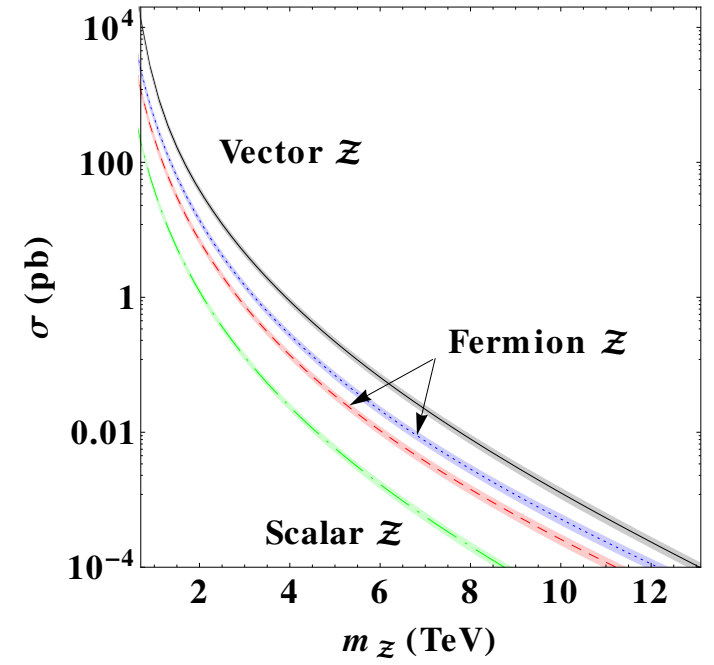

(b)

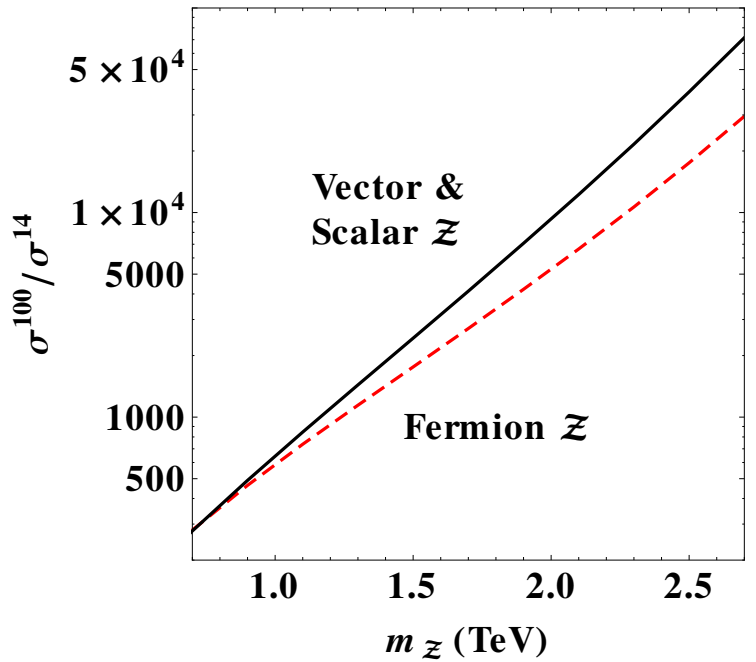

Figure 4. (a) Production cross sections for $p p \rightarrow \mathcal{Z} \overline{\mathcal{Z}}$ at a $p p$ collider with $\sqrt{s}=100 \mathrm{TeV}$ and (b) the cross-section ratios $\sigma(100 \mathrm{TeV}) / \sigma(14 \mathrm{TeV})$, as a function of the mass $m_{\mathcal{Z}}$, for a vector $\mathcal{Z}$ (black solid), Dirac $\mathcal{Z}$ (blue dotted), Majorana $\mathcal{Z}$ (red dashed), and scalar $\mathcal{Z}$ (green dot-dashed). The widths of the bands indicate the estimated theoretical uncertainty [39, 40].

the scalar and fermion cross sections increases at small values of $\beta$, that is, for large values of $m_{\mathcal{Z}}$. The production cross section for Dirac fermions is twice as large as for Majorana fermions, since Dirac fermions have twice the number of independent degrees of freedom. The production rate for a vector $\mathcal{Z}$ is larger than that for Majorana fermions by another factor of about five.

For illustration, figure 4 (a) shows the total production cross sections for $\mathcal{Z} \overline{\mathcal{Z}}$ pairs at a proposed $100-\mathrm{TeV}$ collider. We approximate the $K$-factors for scalar and vector $\mathcal{Z} \overline{\mathcal{Z}}$ production at $\sqrt{s}=100 \mathrm{TeV}$ by assuming the same energy dependence as for the fermionic case, that is, we multiply their $K$-factors at $\sqrt{s}=14 \mathrm{TeV}$ by the ratio of the fermionic $K$-factors at $100 \mathrm{TeV}[39,40]$ and $14 \mathrm{TeV}$. The shaded bands underlying each curve indicate the estimated theoretical uncertainty due to parton distribution functions and the dependence on renormalization and factorization scales, as estimated in refs. [39, 40]. For comparison with the LHC reach, we show the cross-section ratios at the two energies, $\sigma(100 \mathrm{TeV}) / \sigma(14 \mathrm{TeV})$, in figure $4(\mathrm{~b})$. We see that the production cross sections for the color-octet particles could increase by a factor of 500-50,000 for a mass of $1-2.5 \mathrm{TeV}$. Thus, color octets with masses of $\mathcal{O}(10 \mathrm{TeV})$ will become accessible at such a machine, which will reach a cross section of order $0.1-1 \mathrm{fb}$. However, in the following sections, we shall focus on the LHC phenomenology of these particles.

\section{Current bounds from the 8-TeV LHC}

Processes of the form (1.1) can be probed through LHC searches for gluino production with the dominant decay $\tilde{g} \rightarrow t \bar{t} \tilde{\chi}_{1}^{0}$, where $\tilde{\chi}_{1}^{0}$ is the lightest neutralino. In fact, the scenario $\mathrm{vi}(\mathrm{a})+\mathrm{i}$ in table 1 corresponds exactly to this MSSM process. For the other cases in table 1 , 
one can obtain limits by recasting the experimental MSSM results [11-18]. Some of the strongest constraints are obtained from searches for multi-jet final states [13, 14, 16-18]. Here, we instead focus on searches with two same-sign leptons in the final state [11, 12, 15], which have a slightly smaller mass reach but significantly less SM background. The reduced background is an important advantage for model discrimination, which will be discussed in section 5. In particular, we adopt the ATLAS analysis from ref. [11], but the more recent paper [12] and the CMS analysis in ref. [15] lead to similar limits.

We have reproduced the simulation of the MSSM signal in ref. [11] using PYTHIA 6.4 [41] and employing the selection cut sets SR1b and SR3b from that analysis. Explicitly, these cuts are defined as follows:

Pre-sel.: Two leptons with $p_{\mathrm{T}, \ell}>20 \mathrm{GeV},\left|\eta_{e}\right|<2.47,\left|\eta_{\mu}\right|<2.4$, and same charge, $N_{j}$ jets with $p_{\mathrm{T}, j}>40 \mathrm{GeV},\left|\eta_{j}\right|<2.8$,

$N_{b} b$-jets with $70 \% b$-tagging efficiency and $1 \%$ light-jet mis-tagging rate,

$$
\Delta R_{\ell \ell}>0.3, \quad \Delta R_{j j}>0.4, \quad \Delta R_{\ell j}>0.3 .
$$

SR1b: $\quad N_{j} \geq 3, \quad N_{b} \geq 1$,

$$
\left|\boldsymbol{p}_{T}\right|>150 \mathrm{GeV}, \quad M_{T}\left(\ell_{1}, \boldsymbol{p}_{T}\right)>100 \mathrm{GeV}, \quad m_{\mathrm{eff}}>700 \mathrm{GeV} .
$$

SR3b: $\quad N_{j} \geq 4, \quad N_{b} \geq 3$.

Here, $p_{T}$ and $\eta$ denote the transverse momentum and pseudorapidity of an object, respectively, $\Delta R_{a b} \equiv \sqrt{\left(\eta_{a}-\eta_{b}\right)^{2}+\left(\phi_{a}-\phi_{b}\right)^{2}}$, and $\boldsymbol{p}_{T}$ is the missing transverse momentum. The number $N_{j}$ includes both light jets and $b$-jets. The effective mass $m_{\text {eff }}=\sum_{\ell}\left|\mathbf{p}_{T, \ell}\right|+$ $\sum_{j}\left|\mathbf{p}_{T, j}\right|+\left|\mathbf{p}_{T}\right|$ is the scalar sum of the missing transverse momentum and the transverse momenta of the selected leptons and jets, and $M_{T}\left(\ell_{1}, \boldsymbol{p}_{T}\right)=\sqrt{2\left|\mathbf{p}_{T, \ell_{1}}\right|\left|\mathbf{p}_{T}\right|-2 \mathbf{p}_{T, \ell_{1}} \cdot \boldsymbol{p}_{T}}$ is the transverse mass associated with the leading lepton $\ell_{1}$. A cut on $M_{T}$ reduces the background from gauge-boson pair production.

After applying these cuts for $\sqrt{s}=8 \mathrm{TeV}$, we obtain event numbers that are very similar to those in table 5 of ref. [11] (for an MSSM signal using the same gluino, stop and neutralino masses as therein).

The accurate evaluation of the SM backgrounds depends additionally on issues like particle (mis)identification efficiencies; for these, we simply take the numbers from table 3 in ref. [11]. We then combine the SM backgrounds with our simulation of the signal, for the case of the MSSM, which corresponds to scenario vi(a) $+\mathrm{i}$ in table 1 (that is, the fermionscalar-fermion spin combination), and perform a $\chi^{2}$ analysis. The results for $\sqrt{s}=8 \mathrm{TeV}$ are shown in the left panel of figure 5 , as a function of $m_{\mathcal{Z}}$ and $m_{X}$, with $m_{Y}=\left(m_{\mathcal{Z}}+\right.$ $\left.m_{X}\right) / 2$. This sample value of $m_{Y}$ is representative of scenarios in which neither of the $\mathcal{Z}$ and $Y$ decays is near threshold.

As figure 5 shows, fermionic octets (gluinos) decaying into top-quark final states are excluded for $m_{\mathcal{Z}} \lesssim 1160 \mathrm{GeV}$. This limit approximately agrees with ref. [11], although the detailed extent of the excluded region depends on the choice of $m_{Y}$. 

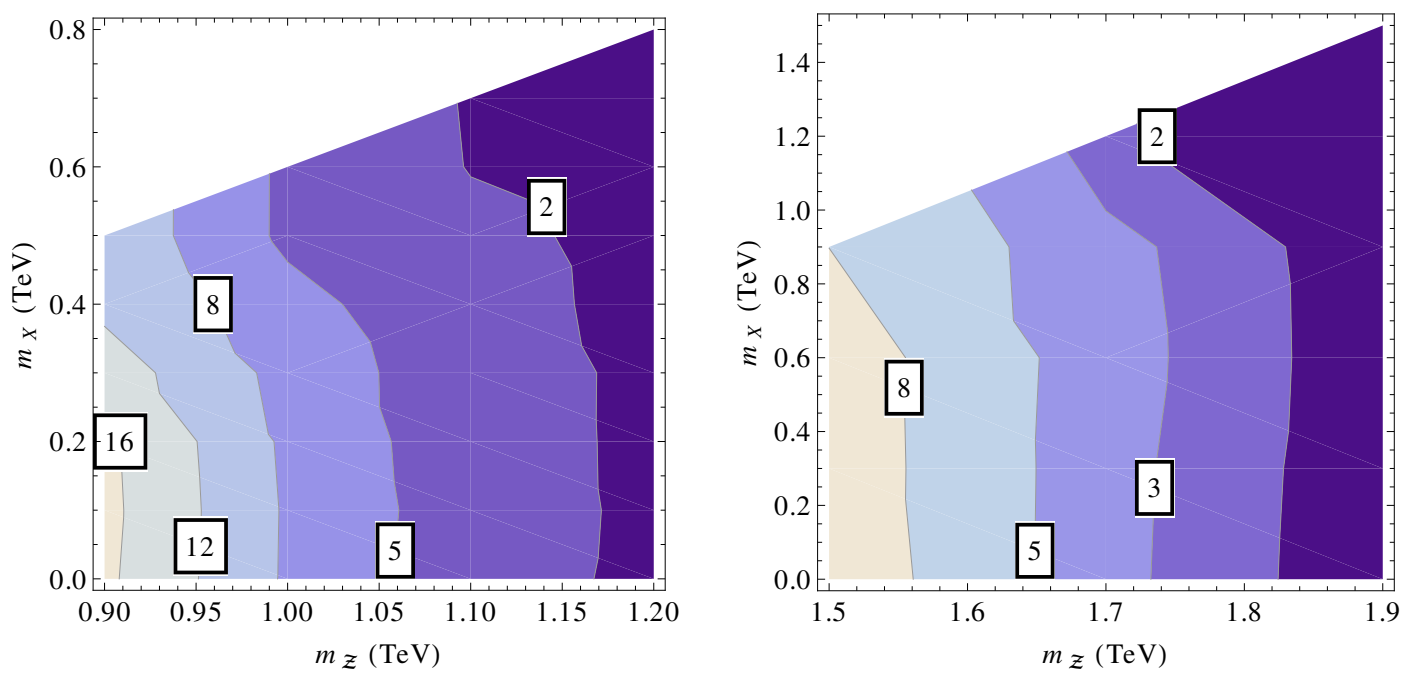

Figure 5. Exclusion limits and projected discovery reach for $p p \rightarrow \mathcal{Z} \overline{\mathcal{Z}} \rightarrow t \bar{t} Y \bar{Y} \rightarrow t \bar{t} t \bar{t} X \bar{X}$ when $\mathcal{Z}$ is fermionic, as a function of the masses of $\mathcal{Z}$ and $X$, with $m_{Y}=\left(m_{\mathcal{Z}}+m_{X}\right) / 2$. The left panel corresponds to $\sqrt{s}=8 \mathrm{TeV}$ and $\mathcal{L}=21 \mathrm{fb}^{-1}$, while the right panel corresponds to $\sqrt{s}=14 \mathrm{TeV}$ and $\mathcal{L}=300 \mathrm{fb}^{-1}$. Contours are labeled with $\sigma$ values indicating the statistical significance.

\section{Signal observability at the 14-TeV LHC}

To obtain projections for $\sqrt{s}=14 \mathrm{TeV}$, we adjust the selection cuts in eqs. (3.1)-(3.3) to obtain roughly the same signal efficiency as for $\sqrt{s}=8 \mathrm{TeV}$. Specifically, all cuts on dimensionless variables are left unchanged, while the cut values for dimensionful variables are scaled up by a factor of $1.1 .^{2}$ We assume that, with this rescaled set of cuts, the same percentage of SM background events is retained as at $\sqrt{s}=8 \mathrm{TeV}$ with the original set of cuts, eqs. (3.1)-(3.3). In other words, we estimate the SM background by scaling the event numbers from ref. [11] by the ratio of the total cross sections for $\sqrt{s}=14 \mathrm{TeV}$ and $\sqrt{s}=8 \mathrm{TeV}$. The cross sections for the dominant SM processes, $p p \rightarrow t \bar{t} W, t \bar{t} Z$ and $p p \rightarrow W Z, Z Z$, are taken from refs. [42-44].

Using this procedure, we obtain the estimated reach of the $14-\mathrm{TeV}$ LHC for the fermionscalar-fermion spin combination given in the right panel of figure 5. Our results are consistent with figure 52 in ref. [22], although in that reference a different set of cuts has been used and the scalar $Y$ (stop) has been decoupled (that is, $m_{Y} \rightarrow \infty$ ).

The exclusion limits (for existing $\sqrt{s}=8 \mathrm{TeV}$ data) and projected reach (for $\sqrt{s}=$ $14 \mathrm{TeV}$ ) depend strongly on the spin of the $\mathcal{Z}$, because of its impact on the cross section $\sigma(p p \rightarrow \mathcal{Z Z})$. One can obtain approximate limits for scalar and vector $\mathcal{Z}$ particles by rescaling the results in figure 5 by the relevant ratios of the cross sections shown in figure 3. Here, it is assumed that spin correlations in the decay chain $\mathcal{Z} \rightarrow t \bar{Y} / \bar{t} Y \rightarrow t \bar{t} X$ have a small effect on the experimental selection efficiency, so that they can be neglected. The results are shown in figure 6 . Note that one can obtain limits for the high-luminosity LHC

\footnotetext{
${ }^{2}$ Checking a range of points throughout the parameter space, we find that the signal efficiencies agree to within $10 \%$, which is within the overall uncertainty of our analysis.
} 

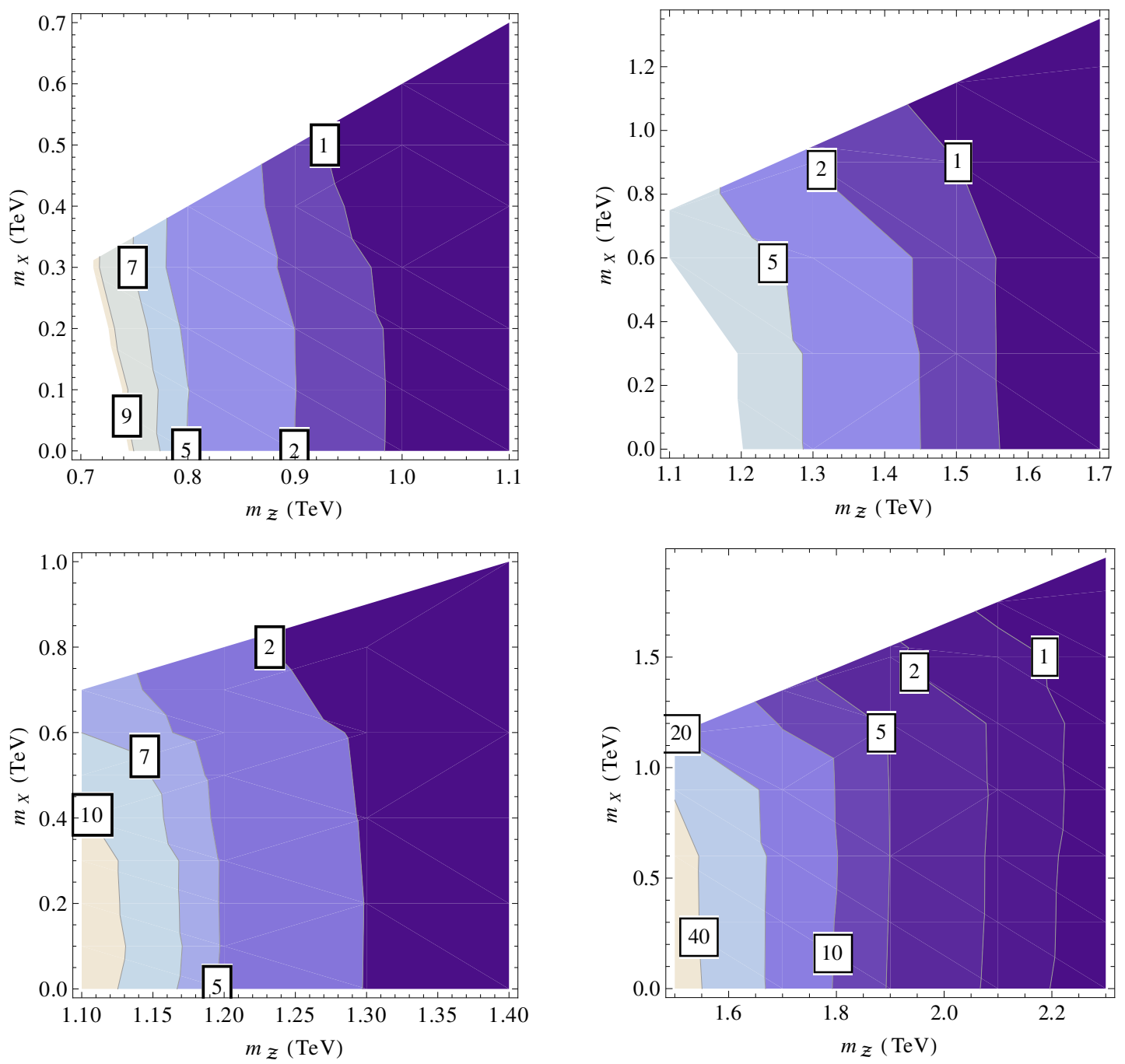

Figure 6. Exclusion limits and projected discovery reach for $p p \rightarrow \mathcal{Z} \overline{\mathcal{Z}} \rightarrow t \bar{t} Y \bar{Y} \rightarrow t \bar{t} t \bar{t} X \bar{X}$ for a scalar (upper) and a vector (lower) $\mathcal{Z}$, as a function of the masses of $\mathcal{Z}$ and $X$, with $m_{Y}=$ $\left(m_{\mathcal{Z}}+m_{X}\right) / 2$. The left panels correspond to $\sqrt{s}=8 \mathrm{TeV}$ and $\mathcal{L}=21 \mathrm{fb}^{-1}$, while the right panels correspond to $\sqrt{s}=14 \mathrm{TeV}$ and $\mathcal{L}=300 \mathrm{fb}^{-1}$. Contours are labeled with $\sigma$ values indicating the statistical significance.

with $\sqrt{s}=14 \mathrm{TeV}$ and $\mathcal{L}=3000 \mathrm{fb}^{-1}$ from the right panels in the figure by rescaling the contours by a factor of $\sqrt{10}$, under the assumption that statistical errors remain dominant.

From figure 6 , one can extract the approximate $2 \sigma$ exclusion limits for scalar and vector $\mathcal{Z}$ production at $8 \mathrm{TeV}$. For a light dark-matter candidate $\left(m_{X} \lesssim 200 \mathrm{GeV}\right)$, the bounds are shown in table 2. The table also lists the expected reach of the $14-\mathrm{TeV}$ run of the LHC for observation of the signal process in eq. (1.1) at the $5 \sigma$ level, again assuming a light dark-matter candidate $\left(m_{X} \lesssim 300 \mathrm{GeV}\right)$.

\section{Determination of model properties}

Once a new-physics signal consistent with the process in eq. (1.1) has been observed at the LHC, it will be crucial to determine the particle properties in order to uncover the 


\begin{tabular}{|c|c|c|c|}
\cline { 2 - 4 } \multicolumn{1}{c|}{} & spin-0 $\mathcal{Z}$ & spin-1/2 & spin-1 \\
\hline $8 \mathrm{TeV}\left(2 \sigma\right.$ with $\left.21 \mathrm{fb}^{-1}\right)$ & $900 \mathrm{GeV}$ & $1160 \mathrm{GeV}$ & $1290 \mathrm{GeV}$ \\
$14 \mathrm{TeV}\left(5 \sigma\right.$ with $\left.300 \mathrm{fb}^{-1}\right)$ & $1280 \mathrm{GeV}$ & $1650 \mathrm{GeV}$ & $1900 \mathrm{GeV}$ \\
$14 \mathrm{TeV}\left(5 \sigma\right.$ with $\left.3000 \mathrm{fb}^{-1}\right)$ & $1480 \mathrm{GeV}$ & $1860 \mathrm{GeV}$ & $2100 \mathrm{GeV}$ \\
\hline
\end{tabular}

Table 2. The $2 \sigma$ exclusion limit at $8 \mathrm{TeV}$ and $5 \sigma$ discovery reach at $14 \mathrm{TeV}$ for a spin- 0 , spin- $1 / 2$ and spin- $1 \mathcal{Z}$, assuming $m_{X} \lesssim 200(300) \mathrm{GeV}$ for $\sqrt{s}=8(14) \mathrm{TeV}$.

underlying theory. The kinematical distributions of the final-state particles can be used to determine the masses, spins and couplings of the $X, Y$ and $\mathcal{Z}$ particles. The analysis of direct pair production of the color triplet $Y, p p \rightarrow Y \bar{Y} \rightarrow t \bar{t} X \bar{X}$, can already yield valuable information about the properties of $Y$ and the singlet $X$ [35]. In this section, we shall instead be concerned primarily with the determination of the properties of the color octet $\mathcal{Z}$ from the process (1.1).

As in the previous sections, we shall focus on the same-sign lepton signature, where each of the directly produced color octets decays through one leptonically and one hadronically

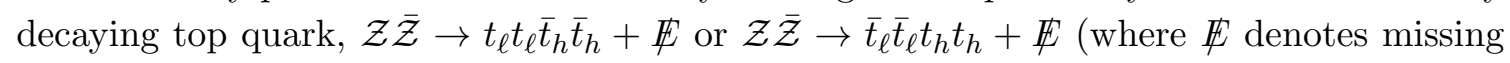
transverse energy). Since this signal has small SM backgrounds, we shall neglect them in the following, in order to highlight more clearly the differences between the various scenarios in table 1. Of course, in a detailed experimental or phenomenological analysis, the SM background contamination and its uncertainty will need to be accounted for, but we leave this for future work.

\subsection{Masses}

The distribution of the invariant mass, $m_{t \bar{t}}$, of the top-antitop pair from the decay chain $\mathcal{Z} \rightarrow t \bar{t} X$ (see figure 1) has a sharp endpoint at

$$
\left(m_{t \bar{t}}^{\max }\right)^{2}=\frac{\left(m_{\mathcal{Z}}^{2}-m_{Y}^{2}\right)\left(m_{Y}^{2}-m_{X}^{2}\right)}{m_{Y}^{2}} .
$$

Even if one of the top quarks decays leptonically, the invariant-mass distribution of the visible $t \bar{t}$ decay products $(b \bar{b} j j \ell)$ still has the endpoint in eq. (5.1), but with a shallower slope. In addition to measuring $m_{t \bar{t}}^{\max }$, one could obtain information about $m_{X}$ and $m_{Y}$ from the process $p p \rightarrow Y \bar{Y} \rightarrow t \bar{t} X \bar{X}$, using the observable $M_{\mathrm{T} 2}$ or one of its variants [35, 4547]. By combining these observables, one could in principle determine $m_{X}, m_{Y}$ and $m_{\mathcal{Z}}$ independently, albeit with poor precision.

If instead one focuses on the all-hadronic decay channel of the top quark, so that all top momenta can be reconstructed, one can take advantage of the kinematical method in ref. [48], which gives relatively large errors in $m_{X}$ but fairly good precision for $m_{\mathcal{Z}}$ and $m_{Y}$. However, the separation of the four top quarks in a given event is a difficult problem, which may be aided by the use of a top-tagging algorithm (see, for example, refs. [49, 50]). Firmer conclusions will require a detailed simulation, which is beyond the scope of this paper. 


\subsection{Spin}

For a decay chain of the form in figure 1 , one can obtain information about the spins of the $\mathcal{Z}, Y$, and $X$ particles from spin-correlation effects, which are reflected in the shape of the $t \bar{t}$ invariant-mass distribution. This strategy has been studied extensively for similar decay chains involving leptons instead of top quarks [51-54]. In contrast to these studies, one must account for the non-negligible mass of the top quark. Secondly, in focusing on the same-sign lepton signature, one cannot fully reconstruct the $t \bar{t}$ mass because of the missing neutrino from the leptonic top decay. Instead, one has to work with the visible decay products of each decay chain, that is, two $b$ jets, two light jets, and one charged lepton $\ell=e, \mu$. The invariant mass of these objects, $m_{b b j j \ell}$, will have a distribution with similar qualitative features to the $m_{t \bar{t}}$ distribution and thus can be used for spin discrimination. Implementing the different spin combinations in table 1 in CALCHEP [55] model files, we have performed parton-level simulations of the decay chain of a single $\mathcal{Z}$ particle, obtaining figure 7 .

For the fermion-scalar-fermion chain (scenarios vi-i), there are no spin correlations between the first and second step of the decay chain in figure 1, so the $m_{t \bar{t}}$ distribution follows the shape dictated by the pure phase-space kinematics. As a result, for this case, $d \Gamma / d m_{b b j j \ell}$ peaks at medium values of $m_{b b j j \ell}$.

In contrast, the scalar-fermion-scalar chain (scenario v-ii) displays maximal correlation effects in the $m_{t \bar{t}}$ distribution, since in this case angular momentum conservation demands alignment between the $t$ and $\bar{t}$ helicities. If the top and anti-top are produced with the same helicity (corresponding to the choices $a_{L}=b_{R}=1, a_{R}=b_{L}=0$ or $\left.a_{L}=b_{R}=0, a_{R}=b_{L}=1\right)$, then they are emitted preferentially in opposite directions, so that their spins add up to zero total angular momentum, as is necessary for the spin-0 initial $\mathcal{Z}$. As a result, in this case the invariant-mass distribution peaks at large values of $m_{b b j j \ell}$. On the other hand, for opposite helicities of the top and anti-top (that is, $a_{L}=b_{L}=1, a_{R}=b_{R}=0$ or $a_{L}=b_{L}=0, a_{R}=b_{R}=1$ ), they are emitted mostly in the same direction and thus the $m_{b b j j \ell}$ distribution peaks at small values.

If $m_{X} / m_{Y} \ll 1$, the results for the scalar-fermion-vector chain (scenario v-iii) are very similar, since the excitation of different spin states of the vector $X$ is suppressed by $m_{X} / m_{Y}$ [52]. On the other hand, for scenario viii-ii or viii-iii (vector-fermion-scalar or vector-fermion-vector), the spin-correlation effects in the $m_{t \bar{t}}$ distribution are slightly reduced, since angular momentum conservation now involves the helicity states of not only the $t$ and $\bar{t}$ but also the parent $\mathcal{Z}$. The correlation effects are even further washed out for scenario vii (fermion-vector-fermion), where both the initial $\mathcal{Z}$ and final $X$ have non-trivial spin states.

To study quantitatively how well one can distinguish between the different spin combinations, we have performed a $\chi^{2}$ analysis for the binned $m_{b b j j \ell}$ distributions, using three bins. $^{3}$ The resulting $\sqrt{\chi^{2}}$ values are shown in table 3 for an assumed signal sample of 857 events. This event yield corresponds to production of Majorana fermion pairs $\mathcal{Z} \overline{\mathcal{Z}}$ with

\footnotetext{
${ }^{3}$ Larger numbers of bins do not yield additional information, but only reduce the discriminative power because of the increased number of degrees of freedom in the statistics.
} 

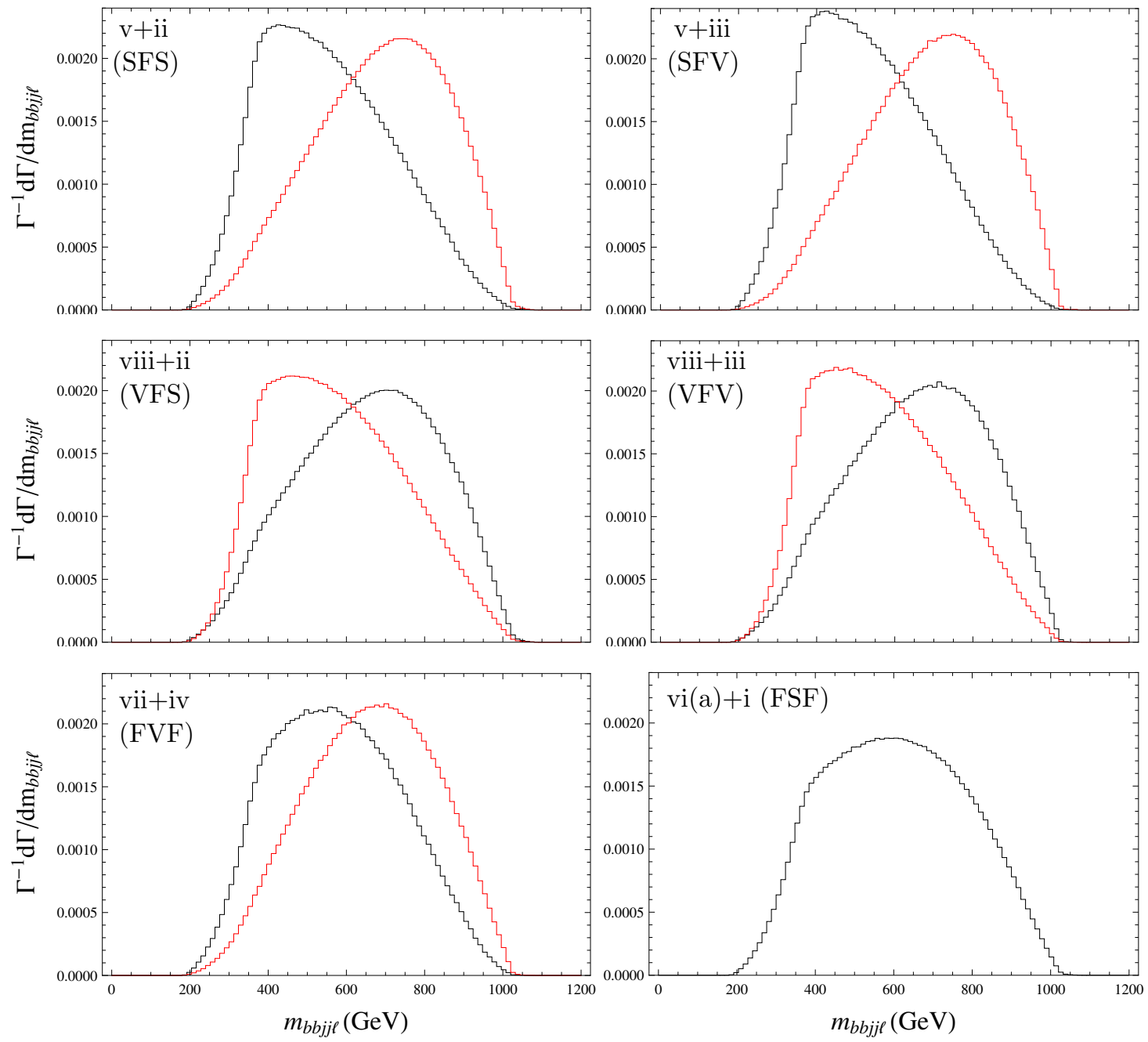

Figure 7. Parton-level invariant-mass distribution of the visible decay products, for the decay chain $\mathcal{Z} \rightarrow t \bar{Y} / \bar{t} Y \rightarrow t \bar{t} X$. The six panels show the results for the scenarios in table 1 , for the two coupling choices $a_{L}=1, a_{R}=0, b_{L}=1, b_{R}=0$ (black) and $a_{L}=0, a_{R}=1, b_{L}=1, b_{R}=0$ (red). Here, S, F, and V denote scalar, fermion, and vector particles, respectively, in the decay chain. The input mass parameters are $m_{\mathcal{Z}}=1200 \mathrm{GeV}, m_{Y}=600 \mathrm{GeV}$ and $m_{X}=100 \mathrm{GeV}$. The distributions have been normalized to unity.

$m_{\mathcal{Z}}=1200 \mathrm{GeV}$ at $\sqrt{s}=14 \mathrm{TeV}$ with an integrated luminosity of $300 \mathrm{fb}^{-1}$. The number of events has been obtained from the simulation results of section 4 and the total cross section in ref. [38].

As table 3 shows, most pairs of spin combinations can be discriminated with high significance. An exception is pairs that differ only in the spin of the invisible $X$. Note that this analysis does not account for detector smearing effects, SM backgrounds, and combinatorial ambiguities in assigning the visible object in a given event to the decay chains of the $\mathcal{Z}$ and $\overline{\mathcal{Z}}$. 


\begin{tabular}{|c|ccccc|}
\cline { 2 - 6 } \multicolumn{1}{c|}{} & \multicolumn{5}{c}{ Spin combinations } \\
\multicolumn{1}{c|}{ SFV } & VFS & VFV & FVF & FSF \\
\hline SFS & 1.3 & 10.3 & 10.6 & 2.4 & 5.3 \\
VFS & & 11.4 & 11.8 & 3.5 & 6.4 \\
VFV & & & 0.35 & 9.6 & 4.7 \\
FVF & & & & 9.6 & 4.8 \\
\hline
\end{tabular}

Table 3. $\sqrt{\chi^{2}}$ values for the discrimination between pairs of different spin combinations, from a binned analysis of the invariant-mass distribution of the visible $t \bar{t}$ decay products. Here, S, F, and $\mathrm{V}$ denote scalar, fermion, and vector particles, respectively, in the decay chain. The results are based on 857 events for the following input mass and coupling parameters: $m_{\mathcal{Z}}=1200 \mathrm{GeV}$, $m_{Y}=600 \mathrm{GeV}, m_{X}=100 \mathrm{GeV} ; a_{L}=1, a_{R}=0, b_{L}=1, b_{R}=0$.

\subsection{Couplings}

The observable invariant-mass distribution depends not only on the spin of the particles in the decay chain but also on the chiral structure of their couplings, that is, whether they are left- or right-handed (see figure 7). Recall that this effect is a manifestation of spin correlations between the two steps of the decay chain, and thus it is absent for a scalar $Y$. Furthermore, the invariant-mass distribution depends only the relative chirality between the first and second interactions in the decay chain, that is, whether $\Gamma$ and $\Gamma^{\prime}$ in table 1 have the same or opposite chirality.

Additional information on the couplings' chirality can be extracted from measurement of the top-quark polarization. The polarization can be determined from the angular distribution of the top-quark decay products. This method is particularly effective for large mass differences $m_{\mathcal{Z}}-m_{Y}$ or $m_{Y}-m_{X}$, when the emitted top quarks are energetic, so their helicity is approximate preserved.

Following the analysis in ref. [35], we study the distribution of the angle $\theta_{b}^{\prime}$ of the $b$ quark with respect to the top-quark boost direction in the top rest frame for the hadronically decaying top, $t_{h}$. Owing to the left-handedness of the weak decay $t \rightarrow W^{+} b$, the $b$ quark is emitted preferentially in the forward direction $\left(\cos \theta_{b}^{\prime}>0\right)$ if the top quark is left-handed, and in the backward direction $\left(\cos \theta_{b}^{\prime}<0\right)$ if the top quark is right-handed.

The resulting $\cos \theta_{b}^{\prime}$ distributions are shown in figure 8 , based on a parton-level simulation with CALCHEP. Since in general it is unknown whether the $t_{h}$ emerged from the first or second step of the decay chain, the observable distributions correspond to an average of both. Consequently, when $\Gamma$ and $\Gamma^{\prime}$ (specified by $a_{L, R}$ and $b_{L, R}$ ) have the same chirality, the $\cos \theta_{b}^{\prime}$ distribution displays a strong polarization signal. On the other hand, if they have opposite chirality, the top quarks from the two decay stages have opposite polarization, leading to an almost flat average $\cos \theta_{b}^{\prime}$ distribution. Thus, the polarization analysis allows one to determine the chirality of the couplings regardless of the spins of $X, Y$ and $\mathcal{Z}$. 

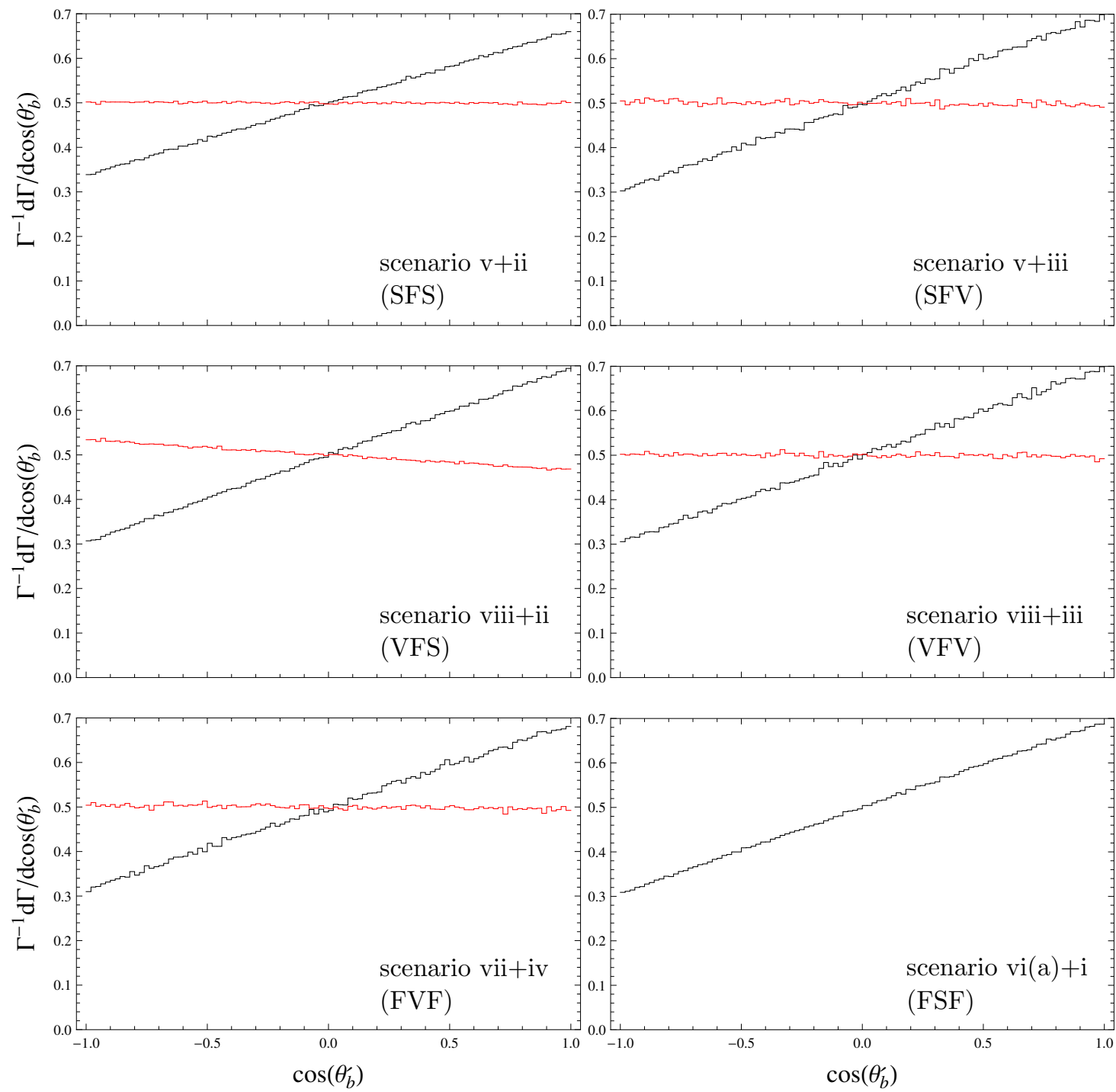

Figure 8. Parton-level angular distribution of the $b$-quark (jet) in the top-quark rest frame, for the decay chain $\mathcal{Z} \rightarrow t \bar{Y} / \bar{t} Y \rightarrow t \bar{t} X$. The six panels show the results for the scenarios in table 1 , for the two coupling choices $a_{L}=1, a_{R}=0, b_{L}=1, b_{R}=0$ (black) and $a_{L}=0, a_{R}=1, b_{L}=1, b_{R}=0$ (red). Here, S, F, and V denote scalar, fermion, and vector particles, respectively, in the decay chain. The input mass parameters are $m_{\mathcal{Z}}=1200 \mathrm{GeV}, m_{Y}=600 \mathrm{GeV}$ and $m_{X}=100 \mathrm{GeV}$. The distributions have been normalized to unity.

\subsection{Distinguishing between Majorana and Dirac particles}

In general, the color-octet $\mathcal{Z}$ field may be self-conjugate or have distinct particles and antiparticles. In this subsection, we investigate whether these two possibilities can be distinguished experimentally at the LHC. For concreteness, we focus on a color octet with spin $1 / 2$, corresponding to a Majorana or Dirac gluino in supersymmetric theories. In broad terms, there are two main approaches to distinguishing between self-conjugate and non-self-conjugate $\mathcal{Z}$ particles: 
1. One can take advantage of the fact that the production cross section for the Dirac case is larger than for the Majorana case by a factor of about 2; see figure 3 and ref. [36]. However, a difficulty associated with this method is that the total cross section also depends strongly on the spin of the $\mathcal{Z}$, its branching fractions and its mass, which is challenging to measure precisely.

2. Alternatively, one can look for characteristics in the decay distributions of the $\mathcal{Z} \overline{\mathcal{Z}}$ pair. This is the approach we study in more detail here.

In the non-self-conjugate (Dirac) case, the $\mathcal{Z}$ (gluino) decays into a $Y$ (stop) and the $\overline{\mathcal{Z}}$ (anti-gluino) decays into a $\bar{Y}$ (anti-stop). For the same-sign lepton signature, this means that one $\ell^{+}$has to come from the second decay step of the $\mathcal{Z}$ and the other from the first decay step of the $\overline{\mathcal{Z}}$ (and vice versa for $\ell^{-}$). In the self-conjugate (Majorana) case, in contrast, the two decay chains are independent, and therefore each of the two same-sign leptons can come from the same stage in its decay chain. Therefore, one expects the energy and $\left|\mathbf{p}_{T}\right|$ distributions of the two leptons to exhibit larger differences - in particular, the $\ell_{2}$ will have a softer distribution - in the Dirac case than in the Majorana case.

The size of this effect depends crucially on kinematics. If $m_{\mathcal{Z}}-m_{Y}$ and $m_{Y}-m_{X}$ are approximately equal, the energy distributions of leptons from the first and second decay steps differ very little, so the Majorana-Dirac distinction is difficult to make. On the other hand, if $m_{\mathcal{Z}}-m_{Y}$ is significantly larger than $m_{Y}-m_{X}$, a lepton emitted from the second decay step $Y \rightarrow t X$ is on average softer then one from the first decay step $\mathcal{Z} \rightarrow \bar{t} Y$, so an attempted discrimination between Majorana and Dirac gluinos is promising. Even in this case, however, the effect is relatively small, so a large integrated luminosity will be needed for this analysis.

To study the effectiveness of this method, we have performed a Monte Carlo simulation of Majorana and Dirac gluino production at the LHC. The simulation of $\mathcal{Z Z}$ (or $\mathcal{Z} \overline{\mathcal{Z}}$ ) pair production with the full decay chain, including top and $W$ decays, with exact matrix elements is very difficult and requires large computational resources. Here, the following simplified approach has been taken: parton-level events for $p p \rightarrow t \bar{t} t \bar{t} X X$ have been generated with CALCHEP and passed to Pythia to perform the top-quark decays. This setup is computationally efficient but ignores the top-quark polarization. Therefore, we have to restrict ourselves to observables that are based only on kinematical features, such as the energy and $\left|\mathbf{p}_{T}\right|$ distributions proposed above.

As concrete examples, we have considered two choices for the mass spectrum:

$$
\begin{array}{llll}
\text { A }: & m_{\mathcal{Z}}=1200 \mathrm{GeV}, & m_{Y}=600 \mathrm{GeV}, & m_{X}=400 \mathrm{GeV} \\
\mathrm{B}: & m_{\mathcal{Z}}=1200 \mathrm{GeV}, & m_{Y}=1000 \mathrm{GeV}, & m_{X}=400 \mathrm{GeV}
\end{array}
$$

In scenario A, $m_{Y}-m_{X} \ll m_{\mathcal{Z}}-m_{Y}$, whereas in scenario $\mathrm{B}, m_{Y}-m_{X} \gg m_{\mathcal{Z}}-m_{Y}$. For the numerical analysis, the same cuts as in section 4 have been applied. With the production cross section for Dirac $\mathcal{Z} \overline{\mathcal{Z}}$ pairs as the reference scenario, this choice produces an event yield of 16,200 for scenario A and 15,970 for scenario B at $\sqrt{s}=14 \mathrm{TeV}$, with an integrated luminosity of $3,000 \mathrm{fb}^{-1}$. 

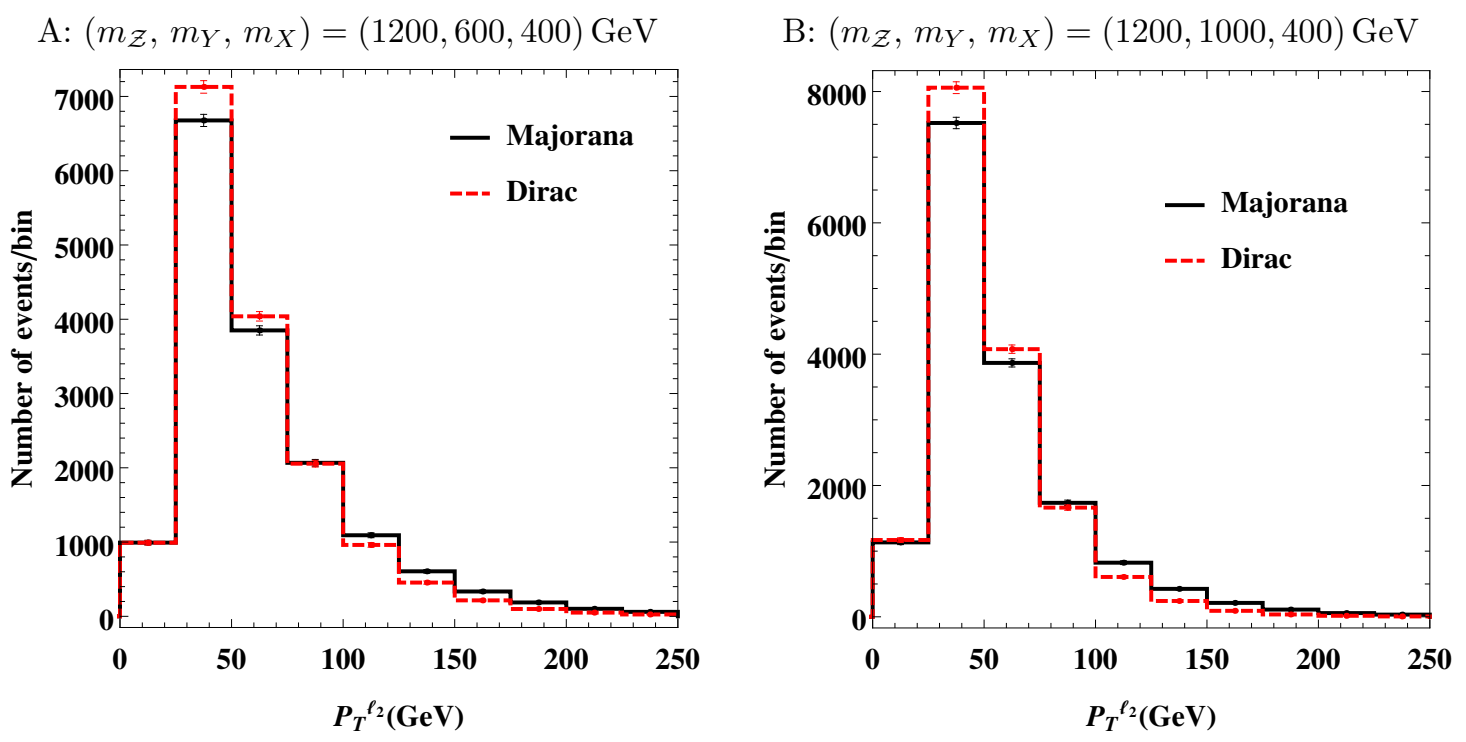

Figure 9. Hadron-level $\left|\mathbf{p}_{T, \ell_{2}}\right|$ distribution from pair production of Majorana fermion pairs $\mathcal{Z Z}$ (black solid) and Dirac fermion pairs $\mathcal{Z} \overline{\mathcal{Z}}$ (red dashed). The Dirac and Majorana cases correspond to scenarios vi(a)+i and vi(b)+i in table 1 , respectively. The chiral couplings have been fixed to $a_{L}=1, a_{R}=0, b_{L}=1, b_{R}=0$. The distributions have been normalized to the production cross section for Dirac octets after application of the selection cuts from section 4 at $\sqrt{s}=14 \mathrm{TeV}$ and $3000 \mathrm{fb}^{-1}\left(N_{\mathrm{ev}}=16200\right.$ and $N_{\mathrm{ev}}=15970$ for scenarios A and B, respectively), and the error bars indicate the statistical uncertainty.

The resulting $\left|\mathbf{p}_{T, \ell_{2}}\right|$ distributions are shown in figure 9. The softer $\left|\mathbf{p}_{T}\right|$ spectrum of the second lepton in the Dirac case can be clearly seen in both scenarios. Performing a binned $\chi^{2}$ analysis with three bins for each distribution, one obtains the following levels of statistical discrimination between Majorana and Dirac octets:

$$
\begin{array}{lll}
300 \mathrm{fb}^{-1}: & \mathrm{A}: \sqrt{\chi^{2}}=3.2, & \text { B }: \sqrt{\chi^{2}}=4.1 ; \\
3000 \mathrm{fb}^{-1}: & \mathrm{A}: \sqrt{\chi^{2}}=10.1, & \text { B }: \sqrt{\chi^{2}}=13.1 .
\end{array}
$$

Thus, a statistically significant exclusion of the scenario not realized in nature may be achievable at the full-energy run of the LHC.

\section{Conclusions}

In this paper, we have introduced a general categorization of new particles motivated by naturalness arguments, with different $\operatorname{spin}(0,1 / 2$, and 1$)$ and color (octet $\mathcal{Z}$, triplet $Y$, and singlet $X)$. There are four possible spin combinations permitting an interaction between the color triplet, singlet and top quark, and four possibilities for a coupling between the octet, triplet and top, as summarized in table 1. The cross sections for the pair production of heavy color-octet particles, $\mathcal{Z} \overline{\mathcal{Z}}$, at LHC energies are shown in figure 3 . These channels would lead to a spectacular signature of four top quarks and missing energy (see eq. (1.1)), where it is assumed that the singlet $X$ is stable and escapes detection. At the $14-\mathrm{TeV}$ 
run of the LHC, this process is observable at the $5 \sigma$ level up to gluon-partner masses of $1280(1480) \mathrm{GeV}$ for a scalar $\mathcal{Z}, 1650(1860) \mathrm{GeV}$ for a fermionic $\mathcal{Z}$, and $1900(2100) \mathrm{GeV}$ for a vector $\mathcal{Z}$ with an integrated luminosity of $300(3000) \mathrm{fb}^{-1}$, provided that the missing particle $X$ (the dark-matter candidate) is not too heavy, that is, $m_{X} \lesssim 300 \mathrm{GeV}$. These results are summarized in table 2 .

If such a signal is discovered, understanding the underlying physics will require the determination of properties of the new particles. As a benchmark, we have taken the typical production rate of Majorana color-octet fermions with $\mathcal{O}(\mathrm{TeV})$ mass at $\sqrt{s}=14 \mathrm{TeV}$ with an integrated luminosity of $300 \mathrm{fb}^{-1}$. Through an analysis of the invariant-mass distribution of the visible decay products of top-quark pairs, most possible spin combinations can be discriminated from each other with high significance; $\sqrt{\chi^{2}}$ values are shown in table 3 . However, pairs that differ only in the spin of the invisible color singlet $X$ are difficult to distinguish.

Furthermore, as figure 7 shows, the observable invariant-mass distribution is also affected by the chiral structure of the couplings of the particles in the decay chain, that is, whether they are left- or right-handed. Additional information on the couplings' chirality can be extracted from the top-quark polarization, which can be determined from the angular distribution of the top-quark decay products. The resulting $\cos \theta_{b}^{\prime}$ distributions are shown in figure 8 . The polarization analysis allows one to determine the chirality of the couplings independently of the spins of $X, Y$ and $\mathcal{Z}$.

Finally, for the case of fermionic color-octet pair production, we have demonstrated that measurements at the LHC also allow us to distinguish whether these particles are Majorana or Dirac fermions, without recourse to the factor of 2 difference in the production cross sections. This is possible because, for a pair of Majorana particles, each can decay randomly and independently into a top quark or antiquark, whereas fermion number is conserved in the decays of a Dirac fermion. Consequently, depending on the mass hierarchy of the new $\mathcal{Z}, Y$ and $X$ particles, there can be distinct differences in the transversemomentum distributions of the final-state decay products, as seen in figure 9 .

We have shown that the full-energy run of the LHC will have a significantly expanded potential for searching for heavy color octets and triplets, as well as identifying their characteristic properties, which could lead to a new understanding of the naturalness of the electroweak scale. At a future 100-TeV VLHC, the mass coverage for a color-octet particle can be substantially extended, with a cross section many orders of magnitude greater than at the LHC, enabling the probing of $\mathcal{Z}$ masses of the order of $10 \mathrm{TeV}$.

\section{Acknowledgments}

This project was supported in part by the National Science Foundation under grant PHY1212635, by the US Department of Energy under grant Nos. DE-FG02-12ER41832 and DE-AC02-98CH10886, and by PITT PACC. Perimeter Institute is supported in part by the Government of Canada through Industry Canada and by the Province of Ontario through the Ministry of Research and Innovation. 
Open Access. This article is distributed under the terms of the Creative Commons Attribution License (CC-BY 4.0), which permits any use, distribution and reproduction in any medium, provided the original author(s) and source are credited.

\section{References}

[1] R. Barbieri and G.F. Giudice, Upper bounds on supersymmetric particle masses, Nucl. Phys. B 306 (1988) 63 [INSPIRE].

[2] S. Dimopoulos and G.F. Giudice, Naturalness constraints in supersymmetric theories with nonuniversal soft terms, Phys. Lett. B 357 (1995) 573 [hep-ph/9507282] [INSPIRE].

[3] M. Papucci, J.T. Ruderman and A. Weiler, Natural SUSY endures, JHEP 09 (2012) 035 [arXiv:1110.6926] [INSPIRE].

[4] S.P. Martin, A supersymmetry primer, Adv. Ser. Direct. High Energy Phys. 21 (2010) 1 [hep-ph/9709356] [INSPIRE].

[5] C.T. Hill and E.H. Simmons, Strong dynamics and electroweak symmetry breaking, Phys. Rept. 381 (2003) 235 [Erratum ibid. 390 (2004) 553] [hep-ph/0203079] [INSPIRE].

[6] C.T. Hill, Topcolor: top quark condensation in a gauge extension of the standard model, Phys. Lett. B 266 (1991) 419 [INSPIRE].

[7] C.T. Hill, Topcolor assisted technicolor, Phys. Lett. B 345 (1995) 483 [hep-ph/9411426] [INSPIRE].

[8] T. Appelquist, H.-C. Cheng and B.A. Dobrescu, Bounds on universal extra dimensions, Phys. Rev. D 64 (2001) 035002 [hep-ph/0012100] [INSPIRE].

[9] B.A. Dobrescu and E. Ponton, Chiral compactification on a square, JHEP 03 (2004) 071 [hep-th/0401032] [INSPIRE].

[10] G. Burdman, B.A. Dobrescu and E. Ponton, Six-dimensional gauge theory on the chiral square, JHEP 02 (2006) 033 [hep-ph/0506334] [INSPIRE].

[11] ATLAS collaboration, Search for strongly produced superpartners in final states with two same sign leptons with the ATLAS detector using $21 \mathrm{fb}^{-1}$ of proton-proton collisions at $\sqrt{s}=8 \mathrm{TeV}$, ATLAS-CONF-2013-007 (2013).

[12] ATLAS collaboration, Search for supersymmetry at $\sqrt{s}=8 \mathrm{TeV}$ in final states with jets and two same-sign leptons or three leptons with the ATLAS detector, JHEP 06 (2014) 035 [arXiv: 1404.2500] [INSPIRE].

[13] ATLAS collaboration, Search for strong production of supersymmetric particles in final states with missing transverse momentum and at least three b-jets using $20.1 \mathrm{fb}^{-1}$ of pp collisions at $\sqrt{s}=8 \mathrm{TeV}$ with the ATLAS detector, ATLAS-CONF-2013-061 (2013).

[14] ATLAS collaboration, Search for new phenomena in final states with large jet multiplicities and missing transverse momentum at $\sqrt{s}=8 \mathrm{TeV}$ proton-proton collisions using the ATLAS experiment, JHEP 10 (2013) 130 [arXiv:1308.1841] [INSPIRE].

[15] CMS collaboration, Search for new physics in events with same-sign dileptons and jets in pp collisions at $\sqrt{s}=8 \mathrm{TeV}$, JHEP 01 (2014) 163 [arXiv:1311.6736] [INSPIRE].

[16] CMS collaboration, Search for supersymmetry in pp collisions at $\sqrt{s}=8 \mathrm{TeV}$ in events with a single lepton, large jet multiplicity and multiple b jets, Phys. Lett. B 733 (2014) 328 [arXiv: 1311.4937] [INSPIRE]. 
[17] CMS collaboration, Search for supersymmetry in pp collisions at $\sqrt{s}=8 \mathrm{TeV}$ in events with three leptons and at least one b-tagged jet, CMS-PAS-SUS-13-008 (2013).

[18] CMS collaboration, Search for new physics in the multijet and missing transverse momentum final state in proton-proton collisions at $\sqrt{s}=8 \mathrm{TeV}$, JHEP 06 (2014) 055 [arXiv: 1402.4770] [INSPIRE].

[19] G.L. Kane, E. Kuflik, R. Lu and L.-T. Wang, Top channel for early SUSY discovery at the LHC, Phys. Rev. D 84 (2011) 095004 [arXiv:1101.1963] [InSPIRE].

[20] R. Essig, E. Izaguirre, J. Kaplan and J.G. Wacker, Heavy flavor simplified models at the LHC, JHEP 01 (2012) 074 [arXiv:1110.6443] [INSPIRE].

[21] Y. Kats, P. Meade, M. Reece and D. Shih, The status of GMSB after 1/fb at the LHC, JHEP 02 (2012) 115 [arXiv: 1110.6444] [INSPIRE].

[22] T. Cohen et al., SUSY simplified models at 14, 33 and 100 TeV proton colliders, JHEP 04 (2014) 117 [arXiv:1311.6480] [INSPIRE].

[23] R.S. Chivukula, A.G. Cohen and E.H. Simmons, New strong interactions at the Tevatron?, Phys. Lett. B 380 (1996) 92 [hep-ph/9603311] [INSPIRE].

[24] Y. Bai and B.A. Dobrescu, Heavy octets and Tevatron signals with three or four b jets, JHEP 07 (2011) 100 [arXiv: 1012 . 5814] [INSPIRE].

[25] R.S. Chivukula, E.H. Simmons and N. Vignaroli, A flavorful top-coloron model, Phys. Rev. D 87 (2013) 075002 [arXiv: 1302.1069] [INSPIRE].

[26] N. Polonsky and S.-f. Su, Low-energy limits of theories with two supersymmetries, Phys. Rev. D 63 (2001) 035007 [hep-ph/0006174] [INSPIRE].

[27] P.J. Fox, A.E. Nelson and N. Weiner, Dirac gaugino masses and supersoft supersymmetry breaking, JHEP 08 (2002) 035 [hep-ph/0206096] [INSPIRE].

[28] M.M. Nojiri et al., Physics beyond the standard model: supersymmetry, arXiv:0802.3672 [INSPIRE].

[29] T. Plehn and T.M.P. Tait, Seeking sgluons, J. Phys. G 36 (2009) 075001 [arXiv:0810.3919] [INSPIRE].

[30] S.Y. Choi et al., Color-octet scalars of $N=2$ supersymmetry at the LHC, Phys. Lett. B 672 (2009) 246 [arXiv: 0812.3586] [INSPIRE].

[31] S.Y. Choi et al., Dirac neutralinos and electroweak scalar bosons of $N=1 / N=2$ hybrid supersymmetry at colliders, JHEP 08 (2010) 025 [arXiv: 1005.0818] [INSPIRE].

[32] W. Kotlarski and J. Kalinowski, Scalar gluons at the LHC, Acta Phys. Polon. B 42 (2011) 2485 [INSPIRE].

[33] W. Kotlarski, A. Kalinowski and J. Kalinowski, Searching for sgluons in the same-sign leptons final state at the LHC, Acta Phys. Polon. B 44 (2013) 2149.

[34] H. Cai, H.-C. Cheng and J. Terning, A spin-1 top quark superpartner, Phys. Rev. Lett. 101 (2008) 171805 [arXiv:0806.0386] [inSPIRE].

[35] C.-Y. Chen, A. Freitas, T. Han and K.S.M. Lee, New physics from the top at the LHC, JHEP 11 (2012) 124 [arXiv: 1207.4794] [INSPIRE].

[36] S.Y. Choi, M. Drees, A. Freitas and P.M. Zerwas, Testing the Majorana nature of gluinos and neutralinos, Phys. Rev. D 78 (2008) 095007 [arXiv:0808.2410] [INSPIRE].

[37] D. Goncalves-Netto, D. Lopez-Val, K. Mawatari, T. Plehn and I. Wigmore, Sgluon pair production to next-to-leading order, Phys. Rev. D 85 (2012) 114024 [arXiv:1203.6358] [INSPIRE]. 
[38] U. Langenfeld, S.-O. Moch and T. Pfoh, QCD threshold corrections for gluino pair production at hadron colliders, JHEP 11 (2012) 070 [arXiv:1208.4281] [INSPIRE].

[39] C. Borschensky, M. Krämer, A. Kulesza, M. Mangano, S. Padhi et al., Squark and gluino production cross sections in pp collisions at $\sqrt{s}=13,14,33$ and $100 \mathrm{TeV}$, Eur. Phys. J. C 74 (2014) 3174 [arXiv: 1407.5066] [inSPIRE].

[40] http://twiki.cern.ch/twiki/bin/view/LHCPhysics/SUSYCrossSections100TeVgluglu.

[41] T. Sjöstrand, S. Mrenna and P.Z. Skands, PYTHIA 6.4 physics and manual, JHEP 05 (2006) 026 [hep-ph/0603175] [INSPIRE].

[42] J.M. Campbell and R.K. Ellis, $t \bar{t} W^{+-}$production and decay at NLO, JHEP 07 (2012) 052 [arXiv: 1204.5678] [INSPIRE].

[43] M.V. Garzelli, A. Kardos, C.G. Papadopoulos and Z. Trócsányi, $t \bar{t} W^{+-}$and $t \bar{t} Z$ hadroproduction at NLO accuracy in QCD with parton shower and hadronization effects, JHEP 11 (2012) 056 [arXiv: 1208.2665] [INSPIRE].

[44] J.M. Campbell, R.K. Ellis and C. Williams, Vector boson pair production at the LHC, JHEP 07 (2011) 018 [arXiv: 1105.0020] [INSPIRE].

[45] C.G. Lester and D.J. Summers, Measuring masses of semiinvisibly decaying particles pair produced at hadron colliders, Phys. Lett. B 463 (1999) 99 [hep-ph/9906349] [INSPIRE].

[46] A.J. Barr and C.G. Lester, A review of the mass measurement techniques proposed for the Large Hadron Collider, J. Phys. G 37 (2010) 123001, see section 4 [arXiv:1004.2732] [INSPIRE].

[47] A.J. Barr et al., Guide to transverse projections and mass-constraining variables, Phys. Rev. D 84 (2011) 095031, see section X [arXiv:1105.2977] [INSPIRE].

[48] H.-C. Cheng, J.F. Gunion, Z. Han, G. Marandella and B. McElrath, Mass determination in SUSY-like events with missing energy, JHEP 12 (2007) 076 [arXiv:0707.0030] [INSPIRE].

[49] D.E. Kaplan, K. Rehermann, M.D. Schwartz and B. Tweedie, Top tagging: a method for identifying boosted hadronically decaying top quarks, Phys. Rev. Lett. 101 (2008) 142001 [arXiv: 0806.0848] [INSPIRE].

[50] T. Plehn, M. Spannowsky, M. Takeuchi and D. Zerwas, Stop reconstruction with tagged tops, JHEP 10 (2010) 078 [arXiv: 1006. 2833] [inSPIRE].

[51] J.M. Smillie and B.R. Webber, Distinguishing spins in supersymmetric and universal extra dimension models at the large hadron collider, JHEP 10 (2005) 069 [hep-ph/0507170] [INSPIRE].

[52] C. Athanasiou, C.G. Lester, J.M. Smillie and B.R. Webber, Distinguishing spins in decay chains at the Large Hadron Collider, JHEP 08 (2006) 055 [hep-ph/0605286] [INSPIRE].

[53] C. Kilic, L.-T. Wang and I. Yavin, On the existence of angular correlations in decays with heavy matter partners, JHEP 05 (2007) 052 [hep-ph/0703085] [INSPIRE].

[54] M. Burns, K. Kong, K.T. Matchev and M. Park, A general method for model-independent measurements of particle spins, couplings and mixing angles in cascade decays with missing energy at hadron colliders, JHEP 10 (2008) 081 [arXiv:0808.2472] [INSPIRE].

[55] A. Belyaev, N.D. Christensen and A. Pukhov, CalcHEP 3.4 for collider physics within and beyond the Standard Model, Comput. Phys. Commun. 184 (2013) 1729 [arXiv:1207.6082] [INSPIRE]. 